\title{
Record Efficiency of Air-Stable Multi-Walled Carbon Nanotube/Silicon Solar Cells
}

\author{
Francesco De Nicola ${ }^{\mathrm{a}, \mathrm{b}, *}$, Matteo Salvato ${ }^{\mathrm{a}, \mathrm{d}}$, Carla Cirillo $^{\mathrm{c}, \mathrm{d}}$, Michele Crivellari ${ }^{\mathrm{e}}$, Maurizio Boscardine ${ }^{\mathrm{e}}$, Manuela \\ Scarselli $^{\mathrm{a}, \mathrm{b}}$, Francesca Nanni ${ }^{\mathrm{f}}$, Ilaria Cacciotti ${ }^{\mathrm{g}}$, Maurizio De Crescenzi ${ }^{\mathrm{a}, \mathrm{b}}$, Paola Castrucci ${ }^{\mathrm{a}, \mathrm{b}}$ \\ ${ }^{a}$ Dipartimento di Fisica, Universitá di Roma Tor Vergata, Via della Ricerca Scientifica 1, 00133 Roma, Italy \\ ${ }^{b}$ Istituto Nazionale di Fisica Nucleare, Universitá di Roma Tor Vergata (INFN-Roma Tor Vergata), Via della Ricerca Scientifica 1, 00133 \\ Roma, Italy \\ ${ }^{c}$ Dipartimento di Fisica, Universitá di Salerno, Via Giovanni Paolo II 132, 84084 Fisciano, Italy \\ ${ }^{d}$ CNR-SPIN Salerno, Universitá di Salerno, Via Giovanni Paolo II 132, 84084 Fisciano, Italy \\ ${ }^{e}$ Fondazione Bruno Kessler (FBK), Via Sommarive 18, 38123 Trento, Italy \\ ${ }^{f}$ Dipartimento di Ingegneria dell'Impresa, Universitá di Roma Tor Vergata (INSTM-UdR Roma Tor Vergata), Via del Politecnico 1, o0133 \\ Roma, Italy \\ ${ }^{g}$ Universitá di Roma Niccoló Cusano (INSTM-UdR), Via Don Carlo Gnocchi 3, 00166 Roma, Italy
}

\begin{abstract}
Multi-walled carbon nanotube (MWCNT) films form efficient heterojunction solar cells with n-type crystalline silicon (n-Si), due to their superior optical and electrical properties. Here, we report air-stable photovoltaic devices with record photoconversion efficiency of $10 \%$. We realized thin films consisting of MWCNTs arranged in semitransparent random networks deposited on n-Si substrates by a simple, rapid, reproducible, and inexpensive vacuum filtration process at room temperature. Such heterojunctions favor high and broadband carrier photogeneration, extending the Si spectral response from near infrared to near ultraviolet range; charge dissociation of ultrafast hot carriers [1]; transport of electrons through n-Si and high-mobility [2] holes through the MWCNT percolative network. Furthermore, by varying the MWCNT film thickness, it is possible tailoring its optical and electrical properties, therefore the overall device optoelectronic features. These results not only pave the way for low-cost, efficient, and broadband photovoltaics, but also are promising for the development of MWCNT-based optoelectronic applications.
\end{abstract}

Keywords: solar cell, photovoltaic, doping, multi-walled carbon nanotube, thin film, silicon, air-stable, record efficiency

\section{Introduction}

In the last decade, the photoconductivity of carbon nanotubes (CNTs) has attracted great interest due to potential applications of their unique optical and electronic properties for the development of novel photosensitive nanomaterials for light sensing and harvesting [3].

Carbon nanotubes may offer a great deal in the development of high-efficiency third-generation solar cells owing to their strong and tunable optical absorption [4, 21];

\footnotetext{
* Corresponding author

Email address: fdenicola@roma2.infn.it (Francesco De

URL: 00390672594532 (Francesco De Nicola)
} Nicola) ultrafast exciton/charge transfer (1-10 ps) [1, 6] 11] extraordinary high mobility $\left(10^{5} \mathrm{~cm}^{2} / \mathrm{Vs}\right)$ [2] simple, rapid, and inexpensive solution-processability [12]. Furthermore, the unique one-dimensional structure of CNTs and the inherent properties of the graphitic $s p^{2}$ carbon lattice allow the fabrication of photovoltaic cells that are highly thermally conductive, mechanical, chemical, and radiation resistant [13]. Moreover, high aspect ratio and flexibility enable CNTs to be weaved into two-dimensional networks with tunable electrical and optical properties, which can be easily deposited on the surface of several materials [12]. In general, heterojunctions [14] offer more choice of materials with appropriate band gaps covering a wider range 
of the solar spectrum, and also potential for the development of a variety of nanomaterial/semiconductor solar cells. So far, a number of researchers have investigated the possibility of utilizing CNT films in devices based on crystalline semiconductors such as Si for heterojunction solar cells (we suggest the review in Ref. [15]). In this framework, on one hand Si photogenerates electron-hole pairs and transports electrons to leads. On the other hand, the CNT film acts both as a photogenerating layer and as holetransporting layer, eventually avoiding metal wiring from the device surface, which shade a portion of incident light, thus replacing the costly indium tin oxide (ITO). In this way, charge generation, separation, transport, and collection can be realized partly by the semi-transparent and conductive CNT thin film itself.

Currently, researcher main efforts have been focused on single-wall carbon nanotube/silicon (SWCNT/Si) heterojunctions, achieving a photoconversion efficiency (PCE) up to $\approx 12 \%[16$ ] without any post-process treatments, up to $\approx 11 \%$ after chemical doping [17, up to $\approx 15 \%$ by adopting single-chirality SWCNTs and gold nanoparticles [18] or relying on antireflective coatings [19], and a record $\approx 17 \%$ by using metal oxide layers [20. However, multiwalled carbon nanotubes (MWCNTs) can efficiently and uniformly absorb more light than SWCNTs from near infrared to ultraviolet [21] because of the absence of an electronic band gap [22 24], thus exhibiting a metallic character, likewise graphene [25]. Moreover, MWCNTs are available at low cost, in large quantity, at lower temperature $\left(<1000{ }^{\circ} \mathrm{C}\right)$ [5] than SWCNTs, and no time-consuming chirality sorting process is needed [18]. Also, the efficiency of MWCNT/Si solar cells can be optimized by tailoring the MWCNT film processing [26] and device post-treatments 27] as for SWCNT/Si devices. Anyway, the potential of MWCNTs has not been fully exploited in photovoltaic applications, indeed MWCNT/Si solar cell PCE has been reported so far to less than $2 \%$ [24, 26, 27]. In particular, one of the primary obstacles in realizing practical appli- cations are the poor operational life-time of CNT/Si devices. Since CNTs are chemically stable, the device degradation typically comes from the Si detrimental oxidation in air [28, 29]. Although, other authors tried to encapsulate 30] the CNT/Si solar cells or to realize air-stable devices [16, 17, 31, 32, their reported PCE values dramatically drop in few time.

In this article, we report air-stable MWCNT/Si heterojunction solar cell devices realized with the process described in the Experimental section. Differently from other authors, our fabrication protocol of MWCNT films is simple, rapid, room temperature, and scalable. Herein, we investigate the optical and electrical properties of the MWCNT film and the whole MWCNT/Si device, by varying the MWCNT film and Si thickness, independently. Therefore, we show that it is possible tailoring in a controlled fashion the PCE of our solar cells up to $10 \pm 1 \%$, which exhibits negligible degradation of PCE up to a month of exposure to air. Hence, our results are 5 times higher than the so far reported PCE values for MWCNT/Si solar cells, and are comparable to the best $\mathrm{PCE}$ values of SWCNT/Si devices in the same conditions (i.e. without any post-process treatments and after chemical doping).

\section{Experimental}

\subsection{Fabrication of CNT films}

Highly pure MWCNT powder (Nanocyl, NC7000, assay 90\%, no amorphous carbon, 10\% metal oxide, outer diameter: $9.5 \mathrm{~nm}$ ) was dispersed in an aqueous solution (30 $\mu \mathrm{g} \mathrm{mL}^{-1}$ ) with $2 \% \mathrm{w} / \mathrm{v}$ sodium dodecyl sulfate (SigmaAldrich, assay $>98.5 \%$ ) anionic surfactant. Surfactant concentration (well above the critical micelle concentration $\approx 0.2 \%$ ) has been calibrated optimizing the solar cell efficiency. In addition, to better disperse the suspension, MWCNTs were tip-ultrasonicated (Branson S250A, $200 \mathrm{~W}, 20 \%$ power, $20 \mathrm{KHz}$ ) in an ice-bath for an hour 
and the unbundled supernatant was collected by pipette. The result was a well-dispersed suspension which is stable for several months. Multi-walled carbon nanotube films were fabricated by a vacuum filtration process of volume aliquots of the dispersion with mixed cellulose ester filters (Pall GN6, 1 in diameter, $0.45 \mu \mathrm{m}$ pore diameter). Subsequently, rinsing in water and in a solution of ethanol, methanol and water (15:15:70) to remove as much surfactant as possible was performed.

\subsection{Device fabrication}

The substrates, provided by Fondazione Bruno Kessler $(\mathrm{FBK})$, are in a top-down configuration. $\mathrm{Au} / \mathrm{Cr}$ ohmic $\left(\mathrm{n}^{+}-\mathrm{Si}\right)$ back contact $(150 \mathrm{~nm})$ was evaporated on n-type crystalline $\mathrm{Si}(100)$ wafers $\left(\rho \approx 3-12 \Omega \mathrm{cm}, N_{D} \approx 6 \cdot 10^{14}\right.$ $\left.\mathrm{cm}^{-3}\right)$ " in diameter, passivated by thermal $\mathrm{SiO}_{2}(300$ $\mathrm{nm})$. The $\mathrm{SiO}_{2}$ layer was patterned in two different ways by a lithographic process with a positive resist followed by a chemical etching in order to obtain a batch of devices with a $2 \times 2 \mathrm{~mm}$ bare $\mathrm{Si}$ window delimited by $\mathrm{SiO}_{2}$. Therefore, the device active area is $0.04 \mathrm{~cm}^{2}$. The Si wafers were made of different thickness $(54-200 \mu \mathrm{m})$ by engraving the crystal only underneath the active area. An $\mathrm{Au} / \mathrm{Cr}$ front contact electrode frame $(150 \mathrm{~nm})$ was then evaporated on $\mathrm{SiO}_{2}$ by masking the $\mathrm{Si}$ active area. Wafers were then cut in squares $1 \times 1 \mathrm{~cm}$. Multi-walled carbon nanotube films were cut and deposited by dry-transfer printing as in Ref. [21, 33] both on HF etched Si active windows and on Carlo Erba soda-lime glass slides for the optical and electrical characterization.

\subsection{Sample characterization}

Optical spectroscopy (Perkin-Elmer Lambda 19 UV/VIS/NIR) was performed to characterize the thickness and the transmittance of MWCNT films. We estimated the film thickness $\tau$ through the Beer-Lambert law. For instance, we used the absorption constant value $\alpha(550 \mathrm{~nm})=(7.4 \pm 0.5) \cdot 10^{5} \mathrm{~cm}^{-1}[21$. As previously reported [12], there is a linear dependence between the thickness of the obtained carbon nanotube film and the aliquot volume $V$ of the same dispersion. Since $V=m / c$, where $m$ is the MWCNT mass in the aliquot and $c$ the concentration of the dispersion, the MWCNT film thickness can be controlled suitably varying aliquot volume and/or dispersion concentration. Multi-walled carbon nanotube microstructure was observed by means of fieldemission scanning electron microscope (FEG-SEM, Leo Supra 35). The acquired micrographs were analyzed with an image software. The obtained MWCNT films appear as homogeneous and porous (void fraction 60\%) random networks, as evident from Figure 17,b. X-ray photoelectron spectroscopy (XPS) and Energy Dispersive X-ray (EDX) analysis on our CNT films showed no trace of $\mathrm{S}$ and $\mathrm{Na}$ due to the SDS surfactant nor of any other metal, within the experimental limit $\approx 1 \%$ of the two techniques 34]. Therefore, our MWCNT films are highly pure and surfactant-free. Electrical measurements were conducted with a Keithley 2602A digital multimeter interfaced to a PC. Sheet resistance values were determined by van der Pauw method after measuring the electrical resistance at room temperature by standard four probe configuration. The same configuration was used to measure electrical resistance as a function of the temperature in He bath fixing the samples at the tip end of a descendant in thermal contact with a Cernox thermometer. Solar cells were tested using a LOT-Oriel solar simulator under AM $1.5 \mathrm{G}$ spectral illumination of $100 \mathrm{mWcm}^{-2}$ (1 sun). The output power density of the simulator was calibrated using a power meter. External quantum efficiency was measured with a custom optical setup: a LOT-Oriel Xe lamp emits white light passing through an Applied PhotoPhysics 300-1000 nm monochromator controlled by PC. Emerging monochromatic light is focused by a lens and divided by a chopper into two beams refocused by two lenses. One beam is then detected by a Si photodiode and the other impinges on the MWCNT/Si solar cell. 

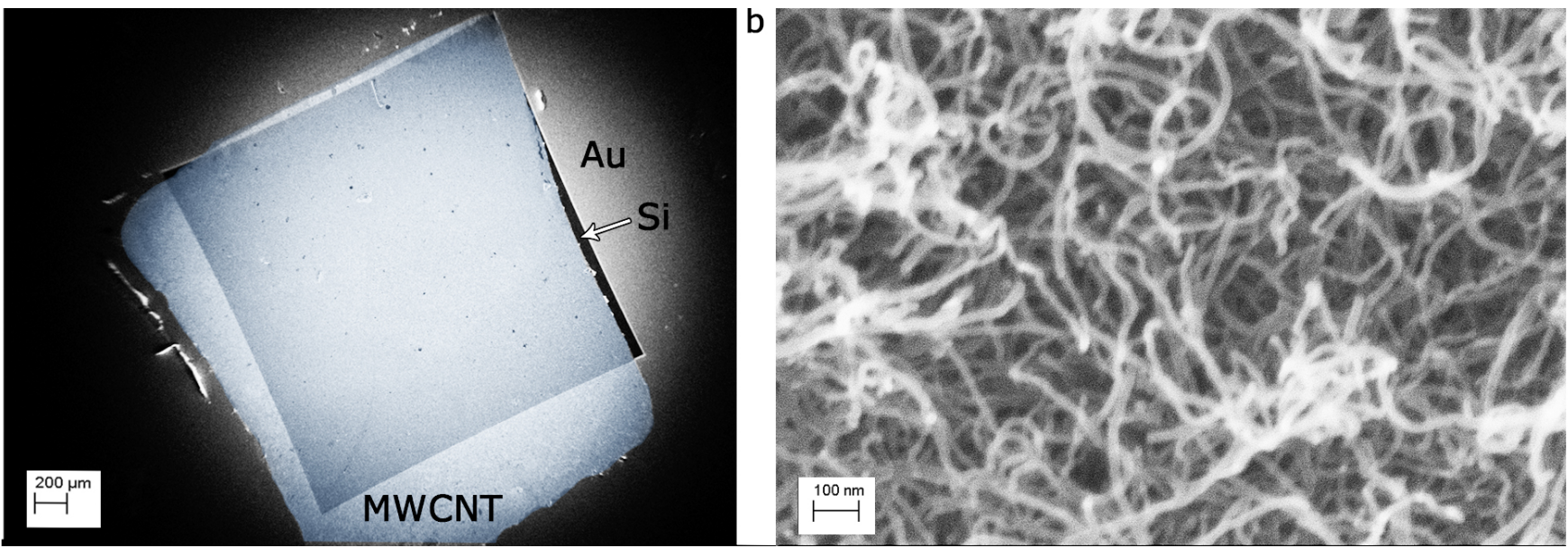

Figure 1: Scanning electron micrographs of a MWCNT film deposited on the solar cell Si substrate magnified at $65 \times(\mathbf{a})$ and $200,000 \times(\mathbf{b})$. a, The MWCNT film (light blue false color) deposited on Si active window (dark square area underneath the film) appears to be homogeneous and defect-free. $\mathbf{b}$, At higher magnification the film exhibits a dense MWCNT random network.

Both AC signals are matched by an Acquire 7265 lock-in connected to a PC.

\section{Results and discussion}

\subsection{MWCNT film electrical and optical properties}

In the following, we introduce our MWCNT film optical and electrical properties. As fitted in Figure $2 a$, the variation of the transmittance $T(\lambda)$ calculated for instance at $550 \mathrm{~nm}$ of MWCNT films with their sheet resistance $R_{s h}$ follows the relation 35

$$
T(\lambda)=\left(1+\frac{Z_{0} \sigma_{a c}(\lambda)}{2 R_{s h} \sigma_{d c}}\right)^{-2},
$$

where $Z_{0}=377 \Omega$ is the free-space impedance and $\sigma_{d c}$, $\sigma_{a c}(\lambda)$ are respectively the electrical and optical conductivity. Since the sheet resistance is related to the film thickness $\tau$ by $R_{s h}=\rho / \tau$ with $\rho$ the MWCNT resistivity (Figure 2 $\mathrm{d}$ ), the thicker the film, the lower the sheet resistance, as well as the transmittance values. Actually, our MWCNT films may have low sheet resistance values with high transmittance, for instance a film $(22.2 \pm 0.4) \mathrm{nm}$ thick has a transmittance $T(550 \mathrm{~nm})=68 \%$ and a sheet resistance $R_{s h}=7 \mathrm{k} \Omega s q^{-1}$. From the fit in Figure $2 \mathrm{~d}$, we report for our MWCNT films $\rho=(1.2 \pm 0.1) \cdot 10^{-5} \Omega \mathrm{m}$, in agreement with the resistivity of bulk graphite 36 .
Furthermore, it is possible to observe in Figure $2 \mathrm{~b}$ the absorbance $A(\lambda)$ of our MWCNT films for different film thicknesses, following the Beer-Lambert law $A(\lambda)=$ $\alpha(\lambda) \tau$, where $\alpha(\lambda)$ is the MWCNT random network absorption coefficient 21]. Moreover, the absorption length $\delta(\lambda)=\alpha(\lambda)^{-1}$ for our MWCNT film compared with that of crystalline $\mathrm{Si}$ is reported in Figure $2 \mathrm{k}$. The material choice in a photovoltaic device is of paramount importance. Indeed, Si is historically the leader material for photovoltaics, owing to its absorption throughout the solar spectrum range deriving from its electronic band gap $(1.12 \mathrm{eV})$. Typically, a $300 \mu \mathrm{m}$ thick $\mathrm{Si}$ wafer is required to absorb all the incident light within $1000 \mathrm{~nm}$ 37. In turn, MWCNT random network films possess an exceptionally high absorption coefficient, which means a very low absorption length. As shown in Figure 2k, just $14 \mathrm{~nm}$ thick MWCNT film is required to attenuate the incident light within $1000 \mathrm{~nm}$ by a factor $1 / e$, a thickness about four orders of magnitude lower than Si. Such a high absorption is due both to their one-dimensional electronic properties and to their random arrangement 21]. On the basis of these results, it is possible fabricating low-cost MWCNT/Si devices by using thin Si wafers, with a huge and broadband absorption. 
The MWCNT film electrical conductance $G$ as a function of its thickness $\tau$ is reported in Figure 2 2 . The plot shown in log scale follows the conductance percolation law 38

$$
G=G_{0}\left(\tau_{c}-\tau\right)^{\mu}
$$

where $G_{0}$ is the conductance of the unity of the percolation network, the thickness $\tau_{c}$ is the conductance percolation threshold, $\mu$ is the universal (i.e. independent of the microscopic details of the system but scale dependent) critical exponent of percolation. Equation 2 is valid when $\tau<\tau_{c}$ and $\tau_{c}-\tau$ is small. The plot states that as the MWCNT film thickness increases beyond the percolation threshold $\tau_{c} \approx 23 \mathrm{~nm}$, the conductance abruptly increases following the power law in equation 2 as conductive paths form. Conversely, below the percolation threshold the material is non-conducting. Fitting our data in log-log scale below the percolation threshold (Figure 2r, inset), we obtain $\mu=1.47 \pm 0.15$, in agreement with the value reported for generic two-dimensional percolative systems $(\mu \approx 1.33)[38$ ] and CNT films 39, 40]. Therefore, the percolation curve shows how to optimize the two-dimensional MWCNT film making it conductive with the minor material amount (the percolation threshold).

\section{2. $M W C N T$ film electronic transport properties}

In order to investigate the conduction mechanism for our MWCNT films, we reported in Figure $2 f$ the normalized conductivity $\sigma(T) / \sigma(300 \mathrm{~K})$ as a function of the temperature $T$ for a MWCNT film with a thickness $\tau=29.3 \pm 0.4$ nm, above the percolation threshold. The observed con-
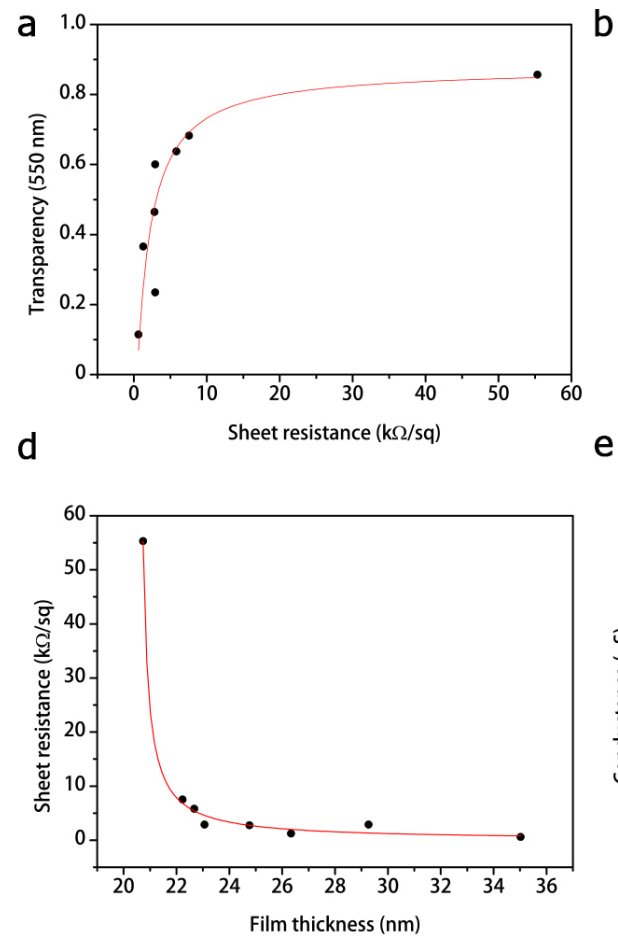
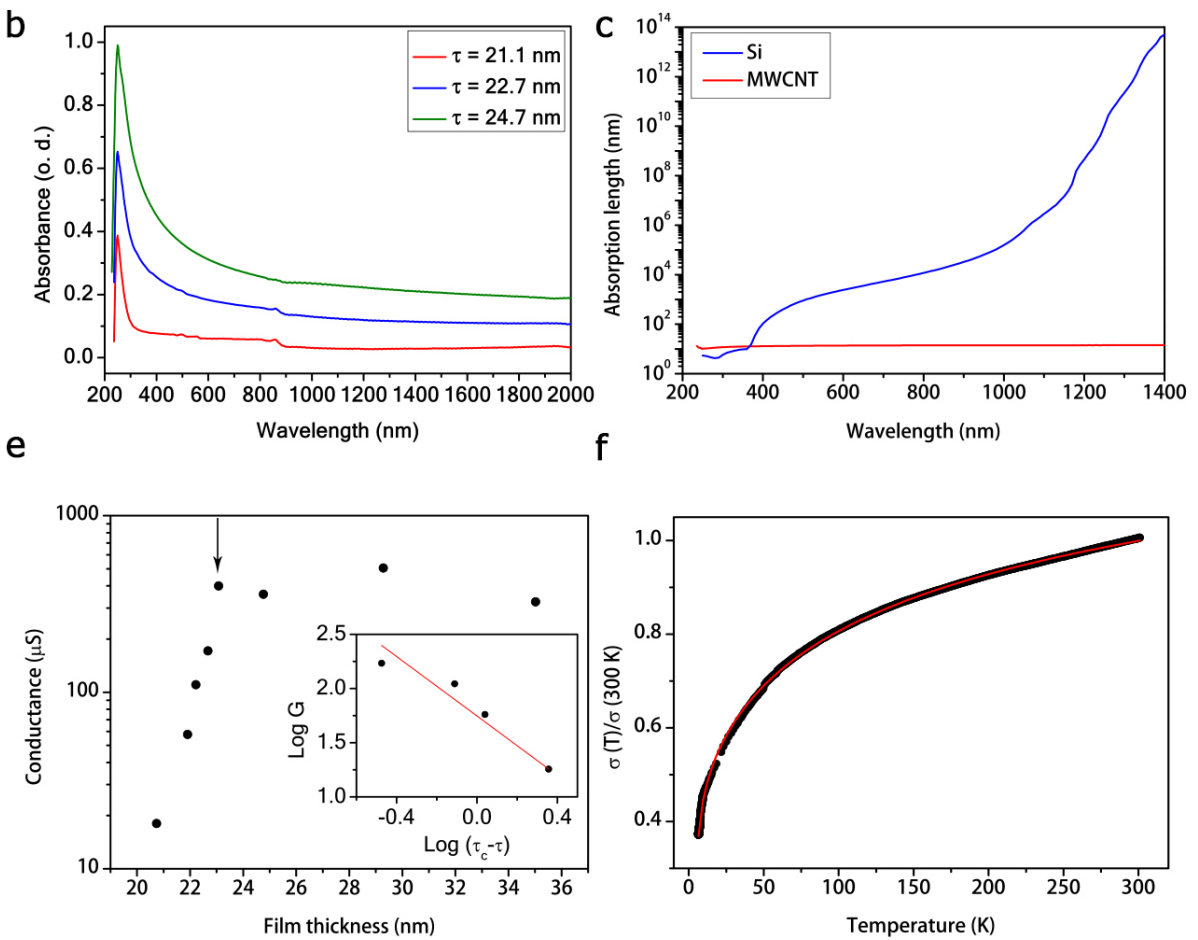

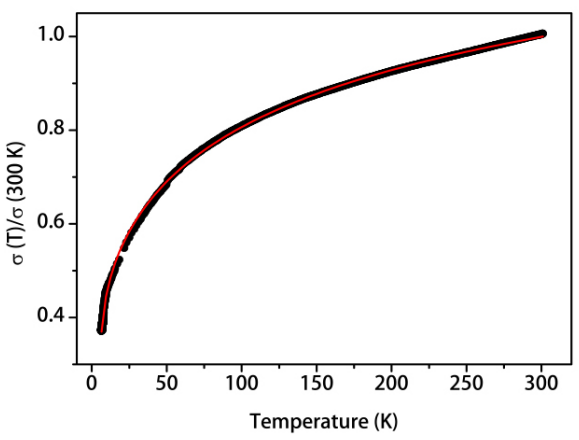

Figure 2: Optical and electrical characterization of the MWCNT film. Transmittance at $550 \mathrm{~nm}$ as a function of the MWCNT film sheet resistance (a). b, Absorbance spectra for different film thicknesses $21.1 \pm 0.3 \mathrm{~nm}$ (red solid curve), $22.7 \pm 0.4$ nm (blue solid curve), and $24.7 \pm 0.4 \mathrm{~nm}$ (green solid curve). c, Absorption length as a function of light wavelength of Si 37] (blue solid line) and our MWCNT films (red solid line). d, Sheet resistance as a function of MWCNT film thickness. e, Electrical conductance of the MWCNT films in log scale as a function of the film thickness. The black arrow marks the percolation threshold $\tau_{c} \approx 23 \mathrm{~nm}$. The log-log plot of the conductance $G$ as a function of the MWCNT film thickness in the inset is fitted by the conductance percolation law for $\tau<\tau_{c}$. $\mathbf{f}$, Normalized conductivity of a $28.7 \pm 0.6 \mathrm{~nm}$ thick MWCNT film as function of temperature. The plot is well fitted by a linear combination of VRH and FIT models. 
a

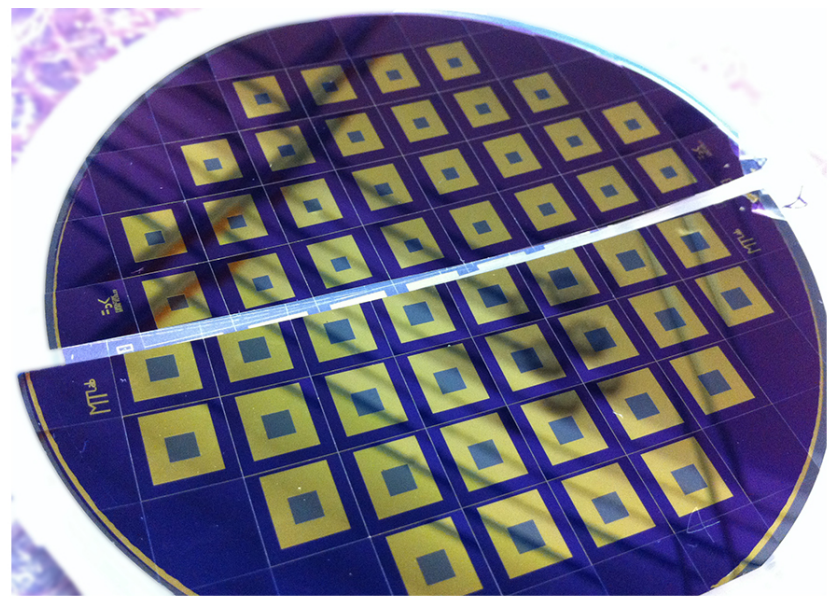

C

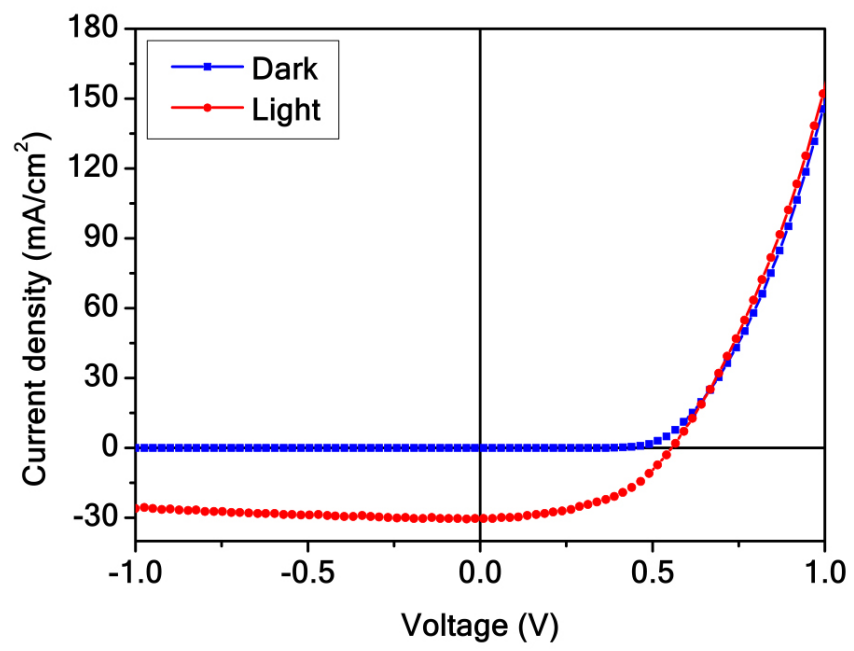

b

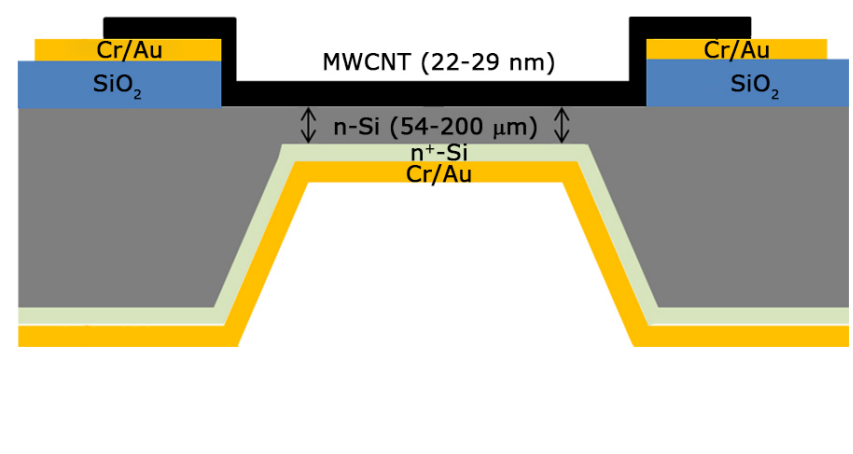

d

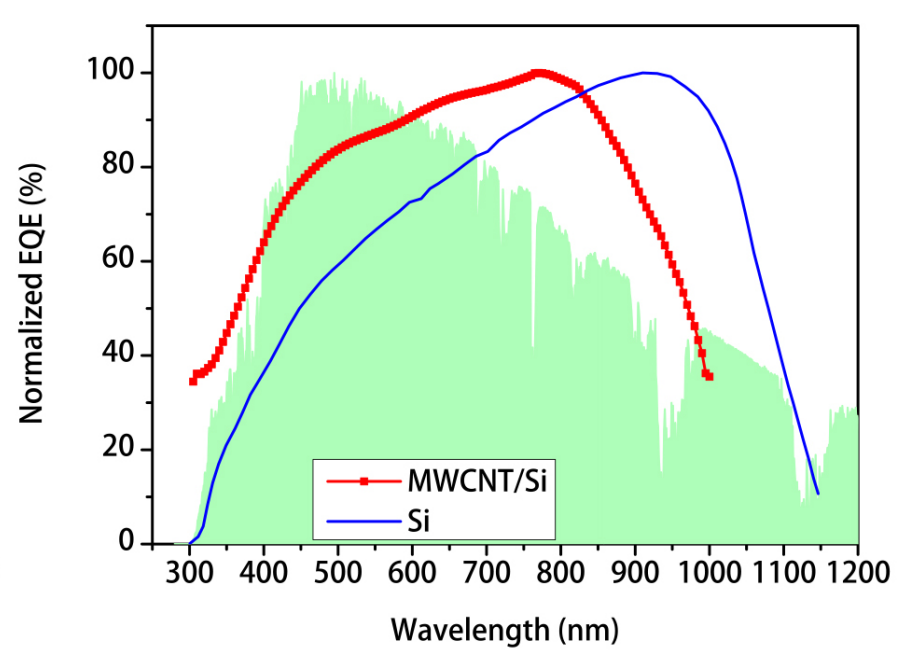

Figure 3: Device characterization. Photographic image of the device before the MWCNT film deposition (a) and sketch in cross section (b) of the MWCNT/Si solar cell. c, Electrical response of our best MWCNT/Si device under dark (blue dotted line) and under illumination (red dotted line). d, External quantum efficiency of our best device (red dotted line) as a comparison with that of a reference p-n Si solar cell (blue solid line) and the AM 1.5 G solar irradiance spectrum (green shaded area). Solar irradiance spectrum and EQEs are normalized to the maximum point.

ductivity tends to zero as the temperature decreases, exhibiting a typical non-metallic behavior. Despite MWCNT films are highly conductive at room temperature, such a trend in conductivity has been reported for individual MWCNTs [1, 42, MWCNT bundles [43, MWCNT films [44, and generally for disordered metals or Fermi glasses, such as conjugated polymer films [45, 46] and amorphous metals [47]. Usually, in those materials disorder and/or Coulomb interactions localize charge carriers at low temperature, inducing a metal/semiconductor tran- sition. We fitted our data with a function, which accounts of a first term denoting the Mott's variable range hopping (VRH) transport [47, and a second term describing the fluctuation-induced tunneling (FIT) transport [48] (see Supplementary Section SI)

$$
\sigma=c_{1} \sigma_{0} e^{-\left(T_{M} / T\right)^{\gamma}}+c_{2} \sigma_{t} e^{\left[T_{1} /\left(T_{0}+T\right)\right]},
$$

with $\sigma_{0}$ the temperature-independent hopping conductivity, $T_{M}$ the Mott's characteristic temperature, $\gamma=$ $1 /(d+1)$ the dimensionality $(d)$ dependent exponent, $\sigma_{t}$ 
the temperature-independent tunneling conductivity, $T_{1}$ the activation temperature required for an electron to tunnel through an energy barrier, and $T_{0}$ the minimum temperature required to activate the thermal conduction to overcome the barrier. The fit returns $\gamma=0.33 \pm 0.01$, which means again that the system is two-dimensional. Furthermore, $c_{1}, c_{2}$ are the normalized weights of the two transport models, which in our case state that the $63 \%$ of transport is by VRH and only $37 \%$ by FIT. Therefore, our MWCNT films can be seen as disordered two-dimensional networks with large high-conductivity clusters separated by small low-conductivity clusters. In such a heterogeneous model [38, 46], weakly localized electrons may percolate by thermally induced hopping between conducting regions, and tunnel through non-conducting regions. This description reasonably supports the fact that MWCNTs are randomly arranged with several intra- and inter-tube contacts, and local defects acting as electronic energy barriers, thus limiting the conduction [46]. Moreover, the presence of low-conductivity barrier clusters in the percolative path means that at frequencies above the semiconductor band gap the $\sigma_{a c}$ will be greater than the $\sigma_{d c}$, as the former is determined by on-tube excitations and not by the inter-tube conductance [38, 40, 46]. Indeed, from the fit in Figure $2 \mathrm{a} \sigma_{a c}(550 \mathrm{~nm}) / \sigma_{d c}=12$.

\section{3. $M W C N T / S i$ solar cells}

Images and sketches of our solar cells, described in detail in the Experimental section, are shown in Figure $3 a, b$. Also, we report in Figure 3 the electrical response of our MWCNT/Si $(22.7 \mathrm{~nm} / 54 \mu \mathrm{m})$ best device in the dark state and under illumination (light state). We achieved an unprecedented $P C E=8 \pm 1 \%$, with a fill factor $F F=49 \pm 7 \%$. Our systematic error on PCE and FF is estimated by the standard deviation over five samples realized in the same way. This small uncertainty demonstrates the high reproducibility of our MWCNT/Si solar cells. From the analysis of the light state curve, we extrapolated the short circuit current density $J_{s c}=30.2 \pm 0.1$ $\mathrm{mA} / \mathrm{cm}^{2}$ and the open circuit voltage $V_{o c}=0.55 \pm 0.01 \mathrm{~V}$, while analyzing the dark state curve (see Supplementary Section SII) we obtained the reverse saturation current density $J_{0}=2.7 \pm 0.2 \cdot 10^{-4} \mathrm{~mA} / \mathrm{cm}^{2}$, the shunt resistance $R_{p}=13.0 \pm 0.2 \mathrm{M} \Omega\left(325 \pm 5 \mathrm{M} \Omega / \mathrm{cm}^{2}\right)$, the series resistance $R_{s}=10.9 \pm 0.1 \Omega\left(272 \pm 2 \Omega / \mathrm{cm}^{2}\right)$, and the diode ideality factor $n=1.80 \pm 0.03$. The extremely high shunt resistance value together with the very low value of reverse saturation current density and series resistance lead to such a high value of PCE, for this kind of devices. Furthermore, the diode ideality factor value close to 2 is symptom of Shockley-Read-Hall (SRH) recombination in the device [37.

In addition, the experimental external quantum efficiency (EQE) spectra for our best MWCNT/Si solar cell in comparison with a p-n Si reference cell (Minolta AK200) and the AM 1.5 G solar spectrum are reported in Figure $3 \mathrm{~d}$. Spectra are normalized to the maximum peak in order to highlight differences in the two devices. It is evident from the plot that our MWCNT/Si cell has a higher efficiency in the visible range, within one standard deviation from the solar spectrum peak. Furthermore, the MWCNT/Si heterojunction has a larger spectral interval of efficiency than the p-n Si cell, ranging from near UV to visible. Also, a large EQE contribution in the NIR above Si band gap due to MWCNT absorption is expected, as it has been reported by other authors [49, 50. We can thus infer that the MWCNT/Si solar cell is a promising device for photovoltaics, with a high and broadband quantum efficiency.

\subsection{Device optimization}

Moreover, optimizing the material thicknesses of the heterojunction is fundamental to achieve high PCE and EQE. In fact, in order to maximize the photogenerated 
carriers collection probability, it is required that the material thicknesses forming the junction are less than the minority carrier diffusion lengths, otherwise carrier recombination is likely to occur inside the material [37. In the present case, the hole diffusion length for $\mathrm{n}-\mathrm{Si}$ $\left(N_{D} \approx 6 \times 10^{14} \mathrm{~cm}^{-3}\right)$ is about $35 \mu \mathrm{m}$, for relaxation time $1 \mu \mathrm{s}$ [37]. Furthermore, since the thicker the material, the more the light is absorbed, there must be a trade-off between the carrier diffusion lengths and the absorption lengths of the materials. We optimized to this purpose the performance of our devices by independently fine tuning the MWCNT film and Si wafer thickness. We report the experimental solar cell electrical response under illumination, EQE, and transient curves as a function of the MWCNT film thickness for a cell with $104 \mu \mathrm{m}$ thick Si wafer (Figure 4 a-c), and as a function of the Si wafer thickness for a cell with $22.7 \pm 0.4 \mathrm{~nm}$ thick MWCNT film (in Figure 4 d-f). We can infer from data that the MWCNT/Si $(22.7 \mathrm{~nm} / 54 \mu \mathrm{m})$ device configuration is the best, since has the highest PCE and EQE. It is worth noting the large variation across the full range of EQE as a function of MWCNT film thickness (Figure $4 \mathrm{~b}$ ), in comparison with the small EQE variation as a function of Si wafer thickness (Figure 4 ). In particular, the change in Si wafer thickness affects more the EQE at long wavelengths, where recombination effects at the back of the device, expected for wafers thicker than the electron diffusion length, compete with higher absorption efficiency. In addition, transient curves in Figure 4 state that the $22.7 \mathrm{~nm}$ thick MWCNT film and the $104 \mu \mathrm{m}$ thick Si wafer lead to the fastest photocurrent response for the solar cell. However, PCE and EQE of the device with $104 \mu \mathrm{m}$ thick Si wafer are significantly lower than those of the device with $54 \mu \mathrm{m}$ thick Si wafer, owing to higher carrier recombination in Si. On the other hand, the thickness change of MWCNT films dramatically affects all the EQE spectrum. We remark that the CNT exciton diffusion length has been reported to be 6-90

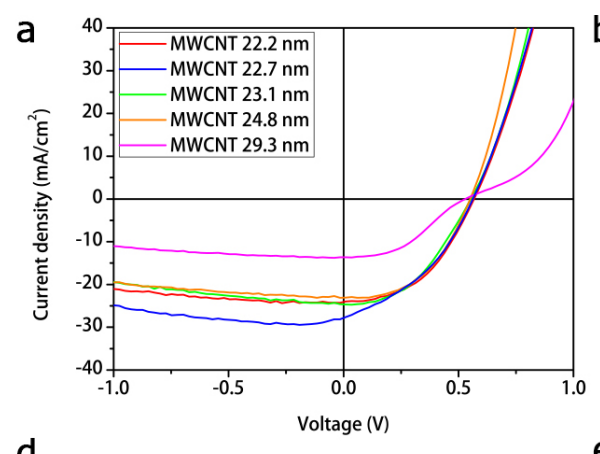

d

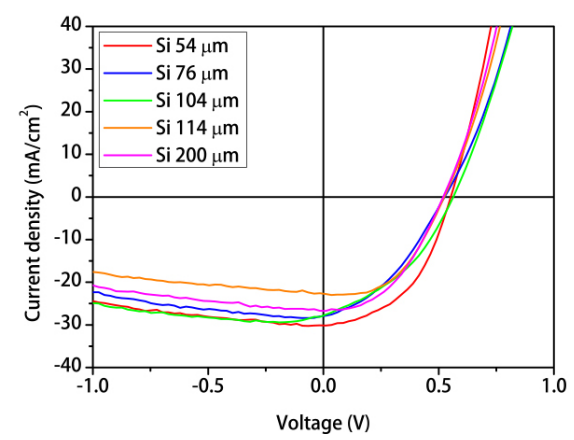

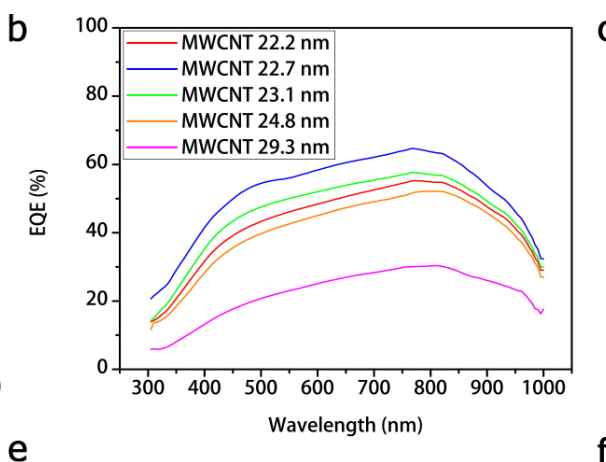

e

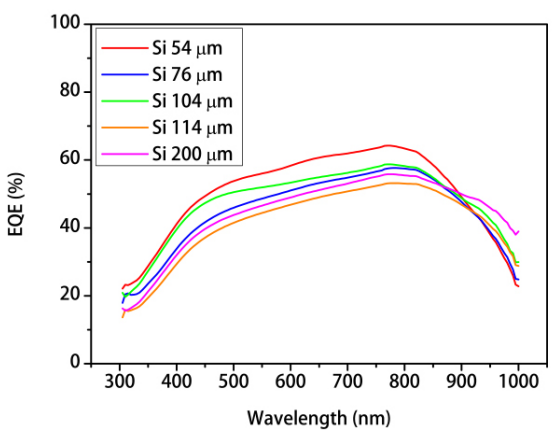

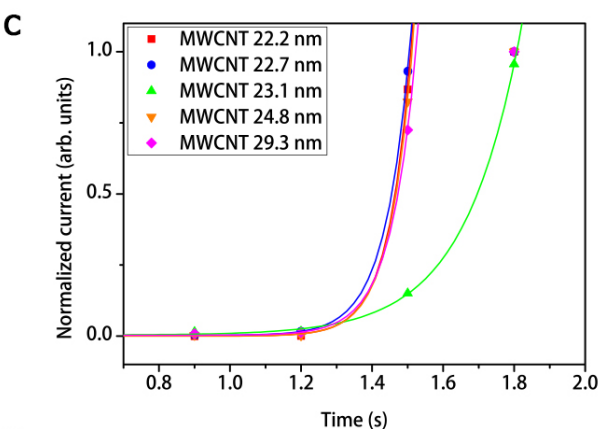

f

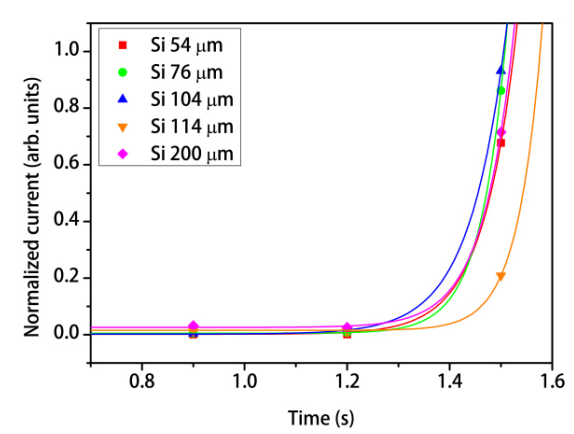

Figure 4: Optimization of the MWCNT/Si device. Electrical response (a), EQE (b), and transient curves (c) of the MWCNT/Si solar cell as a function of the MWCNT film thickness for a cell with $104 \mu \mathrm{m}$ thick Si wafer. Electrical response (d), EQE (e), and transient curves (f) of the MWCNT/Si solar cell as a function of the Si wafer film thickness for a cell with $22.7 \pm 0.4 \mathrm{~nm}$ thick MWCNT film. 
nm [51, 52], consistent with our best MWCNT film thickness observed. Moreover, a film thickness of $27.7 \mathrm{~nm}$ is beyond the aforementioned conductance percolation threshold and absorption length, therefore the MWCNT film is well conducting and can efficiently absorb light and transport the photogenerated minority carriers through the percolative network to collect them at electrodes.

\subsection{Air-stability}

Also, we investigated the stability in time of our MWCNT/Si solar cells (see Supplementary Section SIII for the detailed analysis). It can be observed in Figure $5 \mathrm{a}$ that the PCE of our devices is fairly stable after a month, although they are not encapsulated. In particular, our solar cells reach a maximum of PCE after approximately three days. This fact is due to the formation of an optimal thin $\mathrm{Si}$ oxide layer (1-2 $\mathrm{nm})$ at the $\mathrm{CNT} / \mathrm{Si}$ interface [28, 29, 31]. Differently by other CNT/Si devices reported [16, 17, 30 32, our solar cell PCE does not degrade with time because of $\mathrm{Si}$ oxidation. Actually, the cell is stable after the oxide growth saturates. In particular, it is possible noting in Figure $5 \mathrm{p}$ that the device electrical response, therefore the $\mathrm{PCE}$, fluctuates with time due to small solar simulator intensity fluctuations, which deviate from the fixed value of $100 \mathrm{~mW} / \mathrm{cm}^{2}$ that we used to calculate the PCE. Moreover, also the EQE fluctuates with time but for different reasons, which are still currently under investigation (Figure 5;).

\subsection{Influence of the interfacial silicon oxide layer}

Typically, the device electrical response of the asrealized cells without the thin Si oxide layer exhibits a kink in direct polarization (Figure 5b). It has been reported [53, 54] for organic photovoltaic devices that such a behavior may be due to interfacial effects occurring when large interfacial energy states are present and/or minority carrier mobilities are low or very different. However, it is known that a thin Si oxide layer in Si-based heterojunction is beneficial, because not only it can passivate dangling bonds on the etched Si surface avoiding trapping by surface states, but can also redistribute the electric field in the junction. Generally, in metal-insulator-semiconductor (MIS) heterojunctions [37] the metal high electron density screens the semiconductor charges trapped in oxide interfacial states, developing an interfacial potential $V_{\text {int }}$ that adds up to the built-in potential of the junction. Therefore, in the case of a n-type semiconductor at the metalinsulator interface developing a negative interface charge
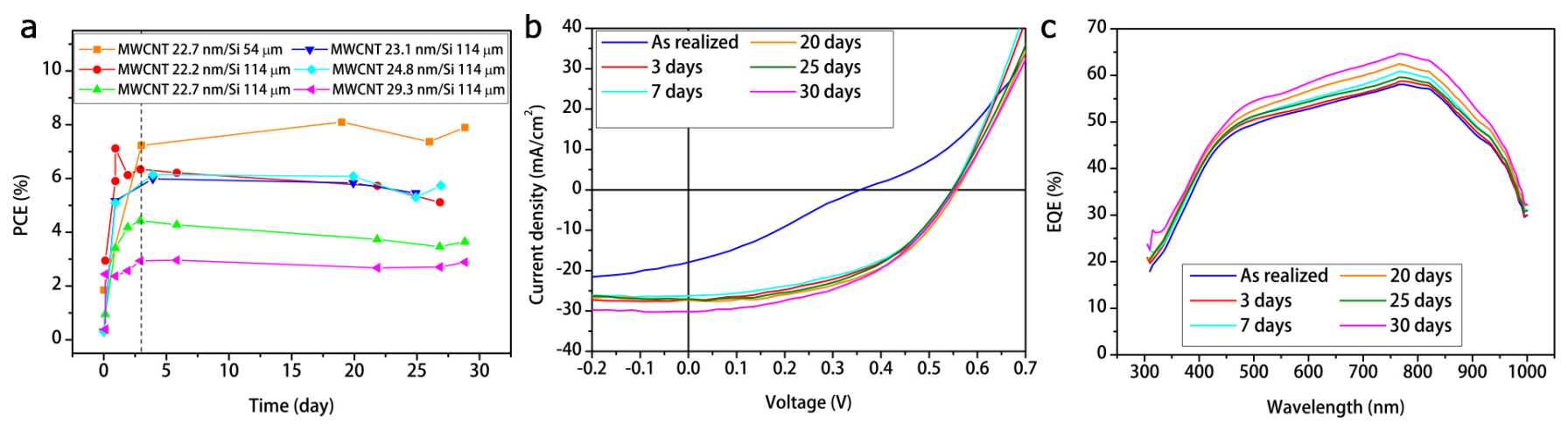

Figure 5: Stability in time of the MWCNT/Si device. a, Photoconversion efficiency of several devices with different MWCNT/Si thickness configurations as a function of the elapsed time. The PCE reaches a maximum after approximately three days, due to the formation of a thin Si oxide layer optimal thickness, then it remains well stable. b, Electrical response as a function of the elapsed time. An as-realized solar cell without Si oxide layer show a kink in direct polarization. c External quantum efficiency of the same solar cell as a function of the elapsed time. 
density $\left(V_{\text {int }}<0\right)$, as in our situation, the built-in potential $V_{b i}$ (i.e. the difference $\Delta \Phi$ between the $\mathrm{Si}$ and MWCNT work functions) should increase owing to $V_{\text {int }}$ following $e V_{b i}=\Delta \Phi-e V_{i n t}$ (see Supplementary Section SIV). Since $V_{b i} \approx V_{o c}$, the higher the built-in potential, the higher the open-circuit voltage, as it is clearly shown in Figure 5b by the $V_{o c}$ difference between an as-realized sample without the oxide presence and the same sample after few days with the oxide layer grown.

\subsection{Doping}

We further investigated the effect of chemical doping the MWCNT/Si solar cell by acid vapors. It has been shown that the PCE of CNT/Si solar cells can be improved by chemical doping with $\mathrm{HNO}_{3}$ vapors or even by wet doping the cells [17, 27-29, 31]. Figure 6a shows the progressive doping effect on a device after several seconds of exposure of the cell front surface to $\mathrm{HNO}_{3}$ vapors. It is clear from the plot that just few seconds of exposure remarkably improve the FF, therefore the PCE of the solar cell from $3 \%$ up to $5 \%$. However, chemical doping is a temporary effect because acids are volatile and quickly photodesorb [55, thus the associated charge transfer is transitory. In fact, since chemical doping is a reversible process, the cell is recovered to its original PCE after only an hour. Conversely, by doping the device with HF vapors, the PCE drops. In addition, upon doping $V_{o c}$ changes from $0.52 \mathrm{~V}$ for the undoped sample to $0.55 \mathrm{~V}$ after $\mathrm{HNO}_{3}$ exposure, down to $0.47 \mathrm{~V}$ after HF exposure. The observed behavior suggests that $\mathrm{HNO}_{3}$ induces a p-type doping in the MWCNT film, while HF a n-type doping (Figure 6f). Therefore, the MWCNT chemical potential $\varepsilon$ is not pinned but can be easily moved by chemical doping, as it occurs in metallic conjugated polymers [45] and SWCNTs [56]. On the basis of this detailed analysis, we achieved for our best MWCNT/Si device shown in Figure 3 . a $25 \%$ relative increment of the $F F$ from $48 \pm 7 \%$ to
$60 \pm 7 \%$, therefore the $P C E$ from $8 \pm 1 \%$ to $10 \pm 1 \%$, after $60 \mathrm{~s}$ of exposure to $\mathrm{HNO}_{3}$ (Figure 7). Such a high value of $\mathrm{PCE}$ is a record for MWCNT/Si solar cells.

In order to offer insight into this doping process of the MWCNT film, we measured the absorption spectrum (Figure 6p) and the sheet resistance of a MWCNT film after several seconds of exposure to $\mathrm{HNO}_{3}$ and $\mathrm{HF}$ vapors. At a first look, the absorption spectrum seems not to vary upon doping. However, by plotting the percent relative absorption (Figure 6p, inset) it is evident that the p-doping effect on the film is to increase the UV-VIS and decrease NIR absorption by few percent, together with a change in sheet resistance $R_{s h}$ up to $30 \%$. The opposite behavior occurs for n-type doping. Our findings suggest a chemical doping of MWCNTs, together with a change in sheet resistance and in absorption (photoconductivity) according to the doping type induced by the oxidizing/reducing agents. We remark that in the absorption spectrum in Figure $6 \mathrm{~b}$ and in the sheet resistance measurements, we did not wait an hour to recover the MWCNT film to the undoped state before exposing it to HF; thus prior to HF doping the film was $\mathrm{HNO}_{3}$ doped for $120 \mathrm{~s}$.

Moreover, another effect of $\mathrm{HNO}_{3}$ (HF) doping is to grow (etch) the thin Si oxide layer at the CNT-Si interface [28, 29, 31]. The induced growth of the Si oxide is shown in Figure 6d. We can distinguish the beneficial effect of the oxide layer from doping, since after a while the PCE does not recover to the undoped state, but it is stable even after several days. In this case, the doping effect is thus given just by the difference between the PCE immediately after doping and after several days, also taking into account the aforementioned PCE fluctuations.

\subsection{Band scheme}

In light of all our results, we can draw the band scheme of our MWCNT/Si heterojunction as depicted in Figure 
8. The band gap $E_{G}$ and the work function $\Phi_{S i}$ of intrinsic $\mathrm{Si}$ are about $1.12 \mathrm{eV}$ and $4.62 \mathrm{eV}$, respectively. In our case, for a dopant concentration of $\approx 6 \cdot 10^{14} \mathrm{~cm}^{-3}$ the quasi-Fermi level is $0.24 \mathrm{eV}$ below the conduction band, therefore our n-Si work function is $\Phi_{n-S i}=4.38 \mathrm{eV}$. The conduction band position in energy of $\mathrm{n}-\mathrm{Si}$, denoted by its electron affinity $\chi=4.06 \mathrm{eV}$, and the valence band lies at $5.17 \mathrm{eV}$. On the other hand, MWCNT film bands are linear dispersed in the reciprocal space with the same mass for electrons and holes. Also, MWCNT bands meet at Dirac points $\left(\Phi_{M W C N T}=4.8 \div 5 \mathrm{eV}\right)$ [23] due to the statistically incidence of at least one metallic chirality in each MWCNT [22, 23]. In chemical doping experiments, we reported for our devices a maximum $V_{o c}=0.55 \mathrm{~V}$. This
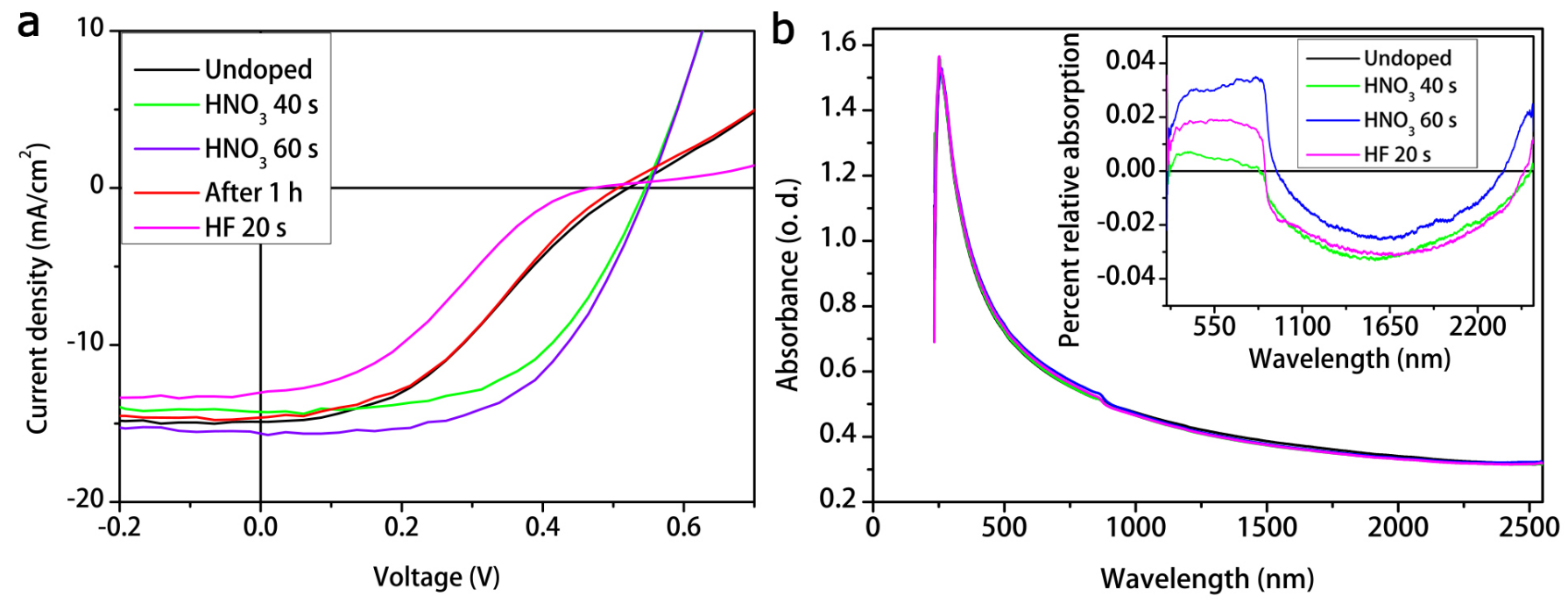

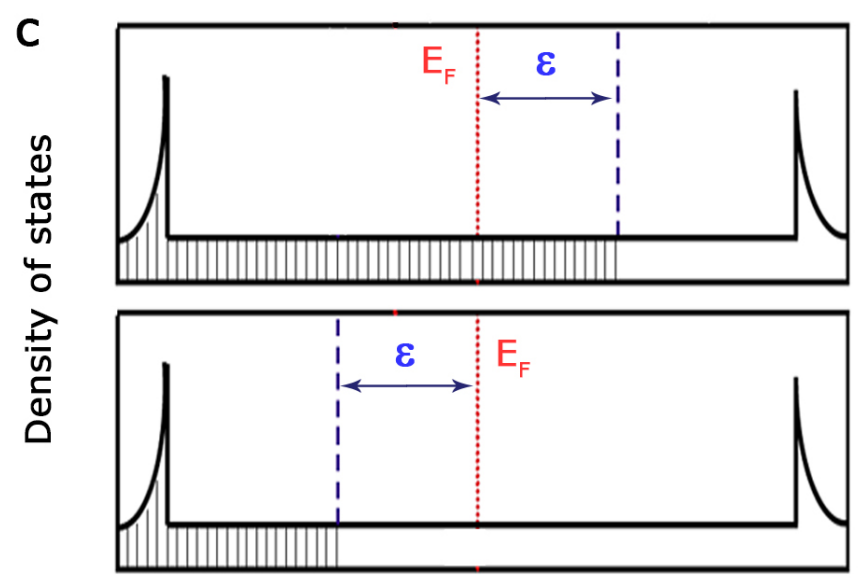

Energy

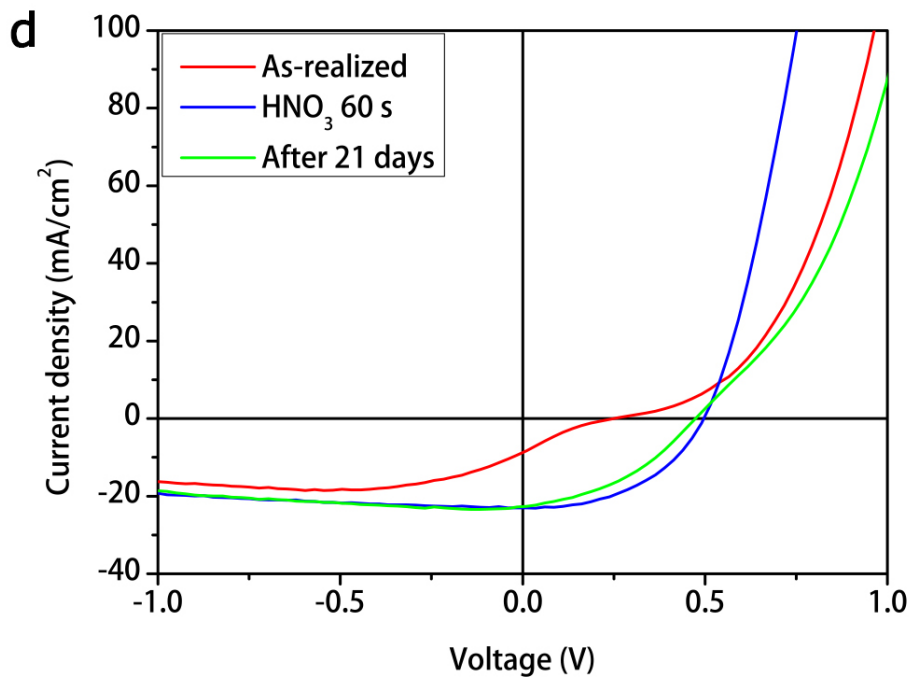

Figure 6: Doping of the MWCNT/Si device. a, Electrical response of a solar cell after chemical doping by $\mathrm{HNO}_{3}$ and $\mathrm{HF}_{\mathrm{F}}$ vapors. $\mathbf{b}$, Absorbance of the same MWCNT film deposited on the cell doped by $\mathrm{HNO}_{3}$ and $\mathrm{HF}$ vapors. inset, MWCNT film percent relative absorption for the undoped sample with sheet resistance $R_{s h}=2.60 \mathrm{k} \Omega \mathrm{sq}^{-1}$ (black solid line), after exposure to $\mathrm{HNO}_{3}$ for $40 \mathrm{~s}$ with $R_{s h}=1.87 \mathrm{k} \mathrm{sqq}^{-1}$ (green curve), after exposure to $\mathrm{HNO}_{3}$ for $60 \mathrm{~s}$ with $R_{s h}=1.82 \mathrm{k} \Omega \mathrm{sq}^{-1}$ (purple curve), after exposure to $\mathrm{HF}$ for $20 \mathrm{~s}$ with $R_{s h}=2.30 \mathrm{k} \Omega \mathrm{sq}-1$ (green curve). c, Scheme of the MWCNT density of state upon chemical doping. Exposure of the film to $\mathrm{HNO}_{3}$ vapors shifts the chemical potential $\varepsilon$ from the Fermi level $E_{F}$ towards the valence band, increasing the hole number (lower panel). Conversely, exposure of the film to HF vapors shifts $\varepsilon$ from $E_{F}$ towards the conductance band, increasing the electron number (upper panel). d, Induced growth of the thin Si oxide layer at the MWCNT-Si interface by $\mathrm{HNO}_{3}$ doping. 


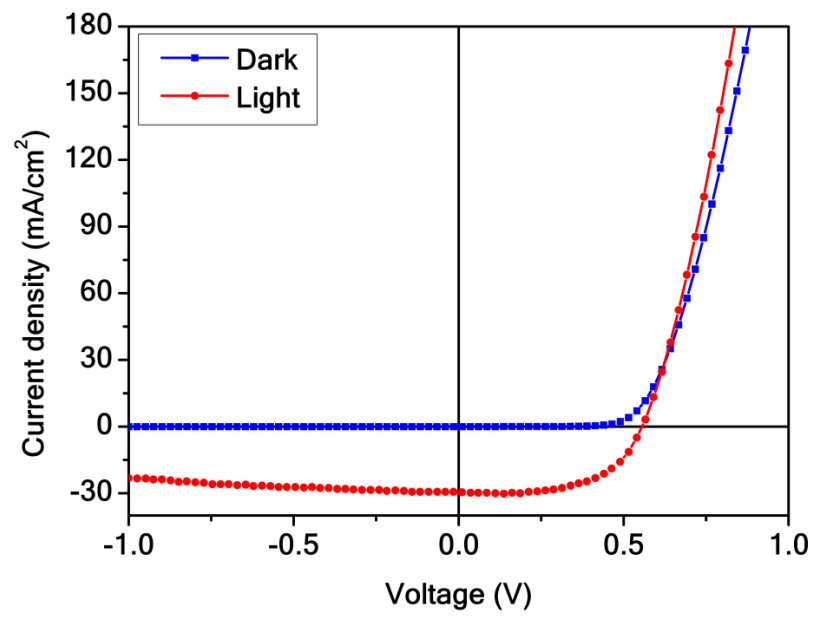

Figure 7: Best MWCNT/Si solar cell in dark (red dotted line) and light (blue dotted line) states after chemical doping by $\mathrm{HNO}_{3}$ vapor exposition for $60 \mathrm{~s}$.

value is approximately the built-in voltage $V_{b i}=0.42 \mathrm{eV}$ established at the heterojunction, without considering the oxide interface potential $V_{i n t}$. Charge separation of photogenerated e-h pairs in both the Si and the MWCNT film occurs due to this built-in field at the interface between the two materials. In particular, in reverse polarization $(V<0)$ hot electrons [1] photogenerated in MWCNTs are ultrafast transferred to the n-type Si region, while hot holes are transported with high mobility [2] to the $\mathrm{Au} / \mathrm{Cr}$ electrode through the MWCNT percolative network (Figure 8). In addition, the thin $\mathrm{SiO}_{2}$ layer redistributes the electric field in the heterojunction so that less of the potential difference is dropped in the thin silicon oxide layer and more in the n-Si [37]. Since the heterojunction Fermi level can be fine tuned by chemical doping, it is not pinned by MWCNT metallic bands, so this kind of heterojunction is not purely a Schottky junction as it has been assumed to be [26, 27] so far, but a sort of MIS.

\section{Conclusion}

In summary, our results represent a proof-of-concept for developing solar cells with high efficiency and reduced cost, by integrating MWCNT and Si technologies. For instance,

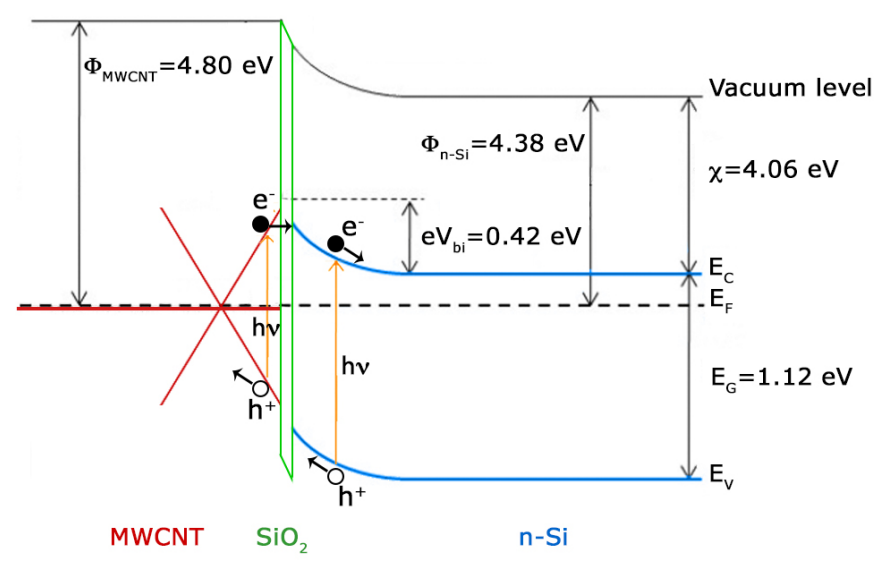

Figure 8: Energetic band scheme of the MWCNT/Si heterojunction in reverse polarization $(V<0)$. The Dirac cone representing MWCNT electronic bands in the reciprocal space has been superimposed on the scheme for clarity.

about $23 \mathrm{~nm}$ thick MWCNT film made by $15 \mu \mathrm{g}$ of MWCNTs, and only $54 \mu \mathrm{m}$ of crystalline $\mathrm{Si}$ are needed to make a heterojunction solar cell with unprecedented $10 \pm 1 \%$ of PCE and $65 \%$ peak EQE. In this way, a reduction of $\mathrm{Si}$ about 6 times with respect to conventional Si p-n solar cells has been obtained for our efficient devices. Finally, our results not only are 5 times higher than the previous reported PCE values of MWCNT/Si devices, but also they are comparable to the best PCE values of SWCNT/Si photovoltaic cells without any post-process treatments and after chemical doping, thus making MWCNT films a valid alternative to SWCNT films.

\section{Acknowledgements}

We thank R. De Angelis, F. De Matteis, and P. Prosposito (Universitá di Roma Tor Vergata, Roma, Italy) for their courtesy of spectrophotometry instrumentation. We thank the European Community for the RISE Project CoExAN GA644076.

[1] G. Galimberti, S. Ponzoni, A. Cartella, M. T. Cole, S. Hofmann, C. Cepek, G. Ferrini, S. Pagliara, Probing the electronic structure of multi-walled carbon nanotubes by transient optical transmittivity, Carbon 57 (2013) 50-58.

[2] T. Dürkop, S. A. Getty, E. Cobas, M. S. Fuhrer, Extraordinary mobility in semiconducting carbon nanotubes, Nano Lett. 4 (2004) 35-39. 
[3] P. Avouris, M. Freitag, V. Perebeinos, Carbon-nanotube photonics and optoelectronics, Nat. Photonics 2 (2008) 341-350.

[4] X. Tu, S. Manohar, A. Jagota, M. Zheng, Dna sequence motifs for structure-specific recognition and separation of carbon nanotubes, Nature 460 (2009) 250-253.

[5] F. De Nicola, P. Castrucci, M. Scarselli, F. Nanni, I. Cacciotti, M. De Crescenzi, Super-hydrophobic multi-walled carbon nanotube coatings for stainless steel, Nanotechnology 26 (2015) 1457011-1457016.

[6] P. H. Tan, A. G. Rozhin, T. Hasan, P. Hu, V. Scardaci, W. I. Milne, A. C. Ferrari, Photoluminescence spectroscopy of carbon nanotube bundles: Evidence for exciton energy transfer, Phys. Rev. Lett. 99 (2007) 1374021-1374024.

[7] J.-H. Han, G. L. C. Paulus, R. Maruyama, D. A. Heller, W.-J. Kim, P. W. Barone, C. Y. Lee, J. H. Choi, M.-H. Ham, C. Song, C. Fantini, M. S. Strano, Exciton antennas and concentrators from core-shell and corrugated carbon nanotube filaments of homogeneous composition, Nat. Mater. 9 (2010) 833-839.

[8] G. Galimberti, S. Pagliara, S. Ponzoni, S. D. Conte, F. Cilento, G. Ferrini, S. Hofmann, M. Arshad, C. Cepek, F. Parmigiani, The photoinduced charge transfer mechanism in aligned and unaligned carbon nanotubes, Carbon 49 (2011) 5246-5252.

[9] R. D. Mehlenbacher, T. J. McDonough, M. Grechko, M.-Y. Wu, M. S. Arnold, M. T. Zanni, Energy transfer pathways in semiconducting carbon nanotubes revealed using two-dimensional white-light spectroscopy, Nat. Commun. 6 (2015) 6732-6739.

[10] S. Ponzoni, G. Galimberti, L. Sangaletti, P. Castrucci, S. D. Gobbo, M. Morbidoni, M. Scarselli, S. Pagliara, Selective optical switching of interface-coupled relaxation dynamics in carbon nanotube-Si heterojunctions, J. Phys. Chem. C 118 (2014) 2411024116 .

[11] M. Grechko, Y. Ye, R. D. Mehlenbacher, T. J. McDonough, M.-Y. Wu, R. M. Jacobberger, M. S. Arnold, M. T. Zanni, Diffusion-assisted photoexcitation transfer in coupled semiconducting carbon nanotube thin films, ACS Nano 8 (2014) 53835394.

[12] F. De Nicola, P. Castrucci, M. Scarselli, F. Nanni, I. Cacciotti, M. De Crescenzi, Multi-fractal hierarchy of single-walled carbon nanotube hydrophobic coatings, Sci. Rep. 5 (2015) 1-9.

[13] A. Jorio, G. Dresselhaus, M. S. Dresselhaus, Carbon Nanotubes Advanced Topics in the Synthesis, Structure, Properties and Applications, Springer, New York, 2008.

[14] J. H. Davies, The Physics of Low-Dimensional Semiconductors An Introduction, Cambridge University Press, Cambridge, 1998.

[15] D. D. Tune, B. S. Flavel, R. Krupke, J. G. Shapter, Carbon nanotube-silicon solar cells, Adv. Energy Mater. 2 (2012) 10431055 .
[16] F. Wang, D. Kozawa, Y. Miyauchi, K. Hiraoka, S. Mouri, Y. Ohno, K. Matsuda, Fabrication of single-walled carbon nanotube/Si heterojunction solar cells with high photovoltaic performance, ACS Photonics 1 (2014) 360-364.

[17] Y. Jia, A. Cao, X. Bai, Z. Li, L. Zhang, N. Guo, J. Wei, K. Wang, H. Zhu, D. Wu, P. M. Ajayan, Achieving high efficiency silicon-carbon nanotube heterojunction solar cells by acid doping, Nano Lett. 11 (2011) 1901-1905.

[18] J. M. Harris, M. R. Semler, S. May, J. A. Fagan, E. K. Hobbie, Nature of record efficiency fluid-processed nanotube-silicon heterojunctions, J. Phys. Chem. C 119 (2015) 10295-10303.

[19] E. Shi, L. Zhang, Z. Li, P. Li, Y. Shang, Y. Jia, J. Wei, K. Wang, H. Zhu, D. Wu, S. Zhang, A. Cao, Tio2-coated carbon nanotube-silicon solar cells with efficiency of $15 \%$, Sci. Rep. 2 (2012) 1-5.

[20] F. Wang, D. Kozawa, Y. Miyauchi, K. Hiraoka, S. Mouri, Y. Ohno, K. Matsuda, Considerably improved photovoltaic performance of carbon nanotube-based solar cells using metal oxide layers, Nat. Commun. 6 (2015) 6305-6312.

[21] F. De Nicola, C. Pintossi, F. Nanni, I. Cacciotti, M. Scarselli, G. Drera, S. Pagliara, L. Sangaletti, M. De Crescenzi, P. Castrucci, Controlling the thickness of carbon nanotube random network films by the estimation of the absorption coefficient, Carbon 95 (2015) 28-33.

[22] R. Saito, Dresselhaus, M. S. Dresselhaus, Electronic structure of double-layer graphene tubules, J. Appl. Phys. 73 (1993) 494500.

[23] W. S. Su, T. C. Leung, C. T. Chan, Work function of singlewalled and multiwalled carbon nanotubes: First-principles study, Phys. Rev. B 76 (2007) 2354131-2354138.

[24] P. Castrucci, C. Scilletta, S. D. Gobbo, M. Scarselli, L. Camilli, M. Simeoni, B. Delley, A. Continenza, M. D. Crescenzi, Light harvesting with multiwall carbon nanotube/silicon heterojunctions, Nanotechnology 22 (2011) 115701-115708.

[25] F. Bonaccorso, Z. Sun, T. Hasan, A. C. Ferrari, Graphene photonics and optoelectronics, Nat. Photonics 4 (2010) 611-622.

[26] Y. Jia, P. Li, J. Wei, A. Cao, K. Wang, C. Li, D. Zhuang, H. Zhu, D. Wu, Carbon nanotube films by filtration for nanotube-silicon heterojunction solar cells, Mater. Res. Bull. 45 (2010) 14011405.

[27] M. Mohammed, Z. Li, J. Cui, T. pin Chen, Acid-doped multiwall carbon nanotube/n-si heterojunctions for enhanced light harvesting, Solar Energy 106 (2014) 171-176.

[28] C. Pintossi, G. Salvinelli, G. Drera, S. Pagliara, L. Sangaletti, S. Del Gobbo, M. Morbidoni, M. Scarselli, M. De Crescenzi, P. Castrucci, Direct evidence of chemically inhomogeneous, nanostructured, Si-O buried interfaces and their effect on the efficiency of carbon nanotube/si photovoltaic heterojunctions, 
J. Phys. Chem. C 117 (2013) 18688-18696.

[29] C. Pintossi, S. Pagliara, G. Drera, F. De Nicola, P. Castrucci, M. De Crescenzi, M. Crivellari, M. Boscardin, L. Sangaletti, Steering the efficiency of carbon nanotube-silicon photovoltaic cells by acid vapor exposure: a real-time spectroscopic tracking, ACS Appl. Mater. Interfaces 7 (2015) 9436-9444.

[30] Y. Jia, P. Li, X. Gui, J. Wei, K. Wang, H. Zhu, D. Wu, L. Zhang, A. Cao, Y. Xu, Encapsulated carbon nanotube-oxide-silicon solar cells with stable 10\% efficiency, Appl. Phys. Lett. 98 (2011) 1331151-1331154.

[31] Y. Jia, A. Cao, F. Kang, P. Li, X. Gui, L. Zhang, E. Shi, J. Wei, K. Wang, H. Zhu, D. Wu, Strong and reversible modulation of carbon nanotube-silicon heterojunction solar cells by an interfacial oxide layer, Phys. Chem. Phys. 14 (2012) 83918396.

[32] K. Cui, A. S. Anisimov, T. Chiba, S. Fujii, H. Kataura, A. G. Nasibulin, S. Chiashi, E. I. Kauppinen, S. Maruyama, Air-stable high-efficiency solar cells with dry-transferred single-walled carbon nanotube films, J. Mater. Chem. A 2 (2014) 11311-11318.

[33] F. De Nicola, P. Castrucci, M. Scarselli, F. Nanni, I. Cacciotti, M. De Crescenzi, Exploiting the hierarchical morphology of single-walled and multi-walled carbon nanotube films for highly hydrophobic coatings, Beilstein J. Nanotechnol. 6 (2015) 353-360.

[34] C Pintossi, G Drera, S Pagliara, and L Sangaletti, Private communication.

[35] M. Dressel, G. Gruner, Electrodynamics of Solids, Optical Properties of Electrons in Matter, Cambridge University Press, Cambridge, 2002.

[36] P. R. Wallace, The band theory of graphite, Phys. Rev. 71.

[37] J. Nelson, The Physics of Solar Cells, Imperial College Press, London, 2003.

[38] M. Sahimi, Applications of Percolation Theory, CRC Press, Boca Raton, 2009.

[39] L. Hu, D. S. Hecht, G. Gruner, Percolation in transparent and conducting carbon nanotube networks, Nano Lett. 4 (2004) 2513-2517.

[40] B. E. Kilbride, J. N. Coleman, J. Fraysse, P. F. F. Cadek, A. Drury, S. Hutzler, S. Roth, W. J. Blau, Experimental observation of scaling laws for alternating current and direct current conductivity in polymer-carbon nanotube composite thin films, J. Appl. Phys. 92 (2002) 4024-4030.

[41] L. Langer, V. Bayot, E. Grivei, J.-P. Issi, J. P. Heremans, C. H. Olk, L. Stockman, C. Van Haesendonck, Y. Bruynseraede, Quantum transport in a multiwalled carbon nanotube, Phys. Rev. Lett. 76 (1996) 479-482.

[42] T. W. Ebbesen, H. J. Lezec, H. Hiura, J. W. Bennett, H. F. Ghaemi, T. Thio, Electrical conductivity of individual carbon nanotubes, Nature 382 (1996) 54-56.
[43] R. Kamalakannan, K. Ganesan, S. Ilango, N. Thirumurugan, V. N. Singh, M. Kamruddin, B. R. Mehta, A. K. Tyagi, The role of structural defects on the transport properties of a fewwalled carbon nanotube networks, Appl. Phys. Lett. 98 (2011) 1921051-1921053.

[44] W. A. de Heer, W. S. Bacsa, A. Châtelain, T. Gerfin, R. Humphrey-Baker, L. Forro, D. Ugarte, Aligned carbon nanotube films: Production and optical and electronic properties, Science 268 (1995) 845-846.

[45] A. J. Heeger, N. S. Sariciftci, E. B. Namdas, Semiconducting and Metallic Polymers, Oxford University Press, Oxford, 2010.

[46] A. B. Kaiser, Electronic transport properties of conducting polymers and carbon nanotubes, Rep. Prog. Phys. 64 (2001) $1-49$.

[47] N. F. Mott, Electrons in disordered structures, Adv. Phys. 16 (1967) 49-144.

[48] P. Sheng, Fluctuation-induced tunneling conduction in disordered materials, Phys. Rev. B 21 (1980) 2180-2195.

[49] M. B. Tzolov, T.-F. Kuo, D. A. Straus, A. Yin, J. Xu, Carbon nanotube-silicon heterojunction arrays and infrared photocurrent responses, J. Phys. Chem. C 111 (2007) 5800-5804.

[50] J. Lehman, A. Sanders, L. Hanssen, B. Wilthan, J. Zeng, C. Jensen, Very black infrared detector from vertically aligned carbon nanotubes and electric-field poling of lithium tantalate, Nano Lett. 10 (2010) 3261-3266.

[51] L. Cognet, D. A. Tsyboulski, J.-D. R. Rocha, C. D. Doyle, J. M. Tour, R. B. Weisman, Stepwise quenching of exciton fluorescence in carbon nanotubes by single-molecule reactions, Science 316 (2007) 1465-1468.

[52] L. Lüer, S. Hoseinkhani, D. Polli, J. Crochet, T. Hertel, Size and mobility of excitons in $(6,5)$ carbon nanotubes, Nat. Physics 5 (2009) 54-58.

[53] B. Y. Finck, B. J. Schwartz, Understanding the origin of the scurve in conjugated polymer/fullerene photovoltaics from driftdiffusion simulations, Appl. Phys. Lett. 103 (2013) 05330610533064 .

[54] W. Tress, A. Petrich, M. Hummert, M. Hein, K. Leo, M. Riede, Imbalanced mobilities causing s-shaped IV curves in planar heterojunction organic solar cells, Appl. Phys. Lett. 98 (2011) 0633011-0633013.

[55] R. J. Chen, N. R. Franklin, J. Kong, J. Cao, T. W. Tombler, Y. Zhang, H. Dai, Molecular photodesorption from single-walled carbon nanotubes, Appl. Phys. Lett. 79 (2001) 2258-2260.

[56] J. L. Blackburn, T. M. Barnes, M. C. Beard, Y.-H. Kim, R. C. Tenent, T. J. McDonald, B. To, T. J. Coutts, M. J. Heben, Transparent conductive single-walled carbon nanotube networks with precisely tunable ratios of semiconducting and metallic nanotubes, ACS Nano 2 (2008) 1266-1274. 